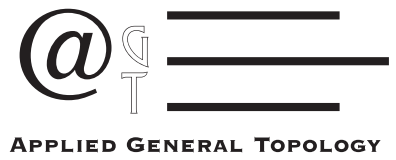

Appl. Gen. Topol. 21, no. 2 (2020), 247-264

doi:10.4995/agt.2020.13049

(C) AGT, UPV, 2020

\title{
Topological distances and geometry over the symmetrized Omega algebra
}

\author{
Mesfer Alqahtani ${ }^{a}$, Cenap Özel $^{a}$ And Hanifa Zekraoui ${ }^{b}$ \\ a Department of Mathematics, King Abdulaziz University, Jeddah, Kingdom of Saudi Ara- \\ bia (mesfer_alqhtani@hotmail.com, cozel@kau.edu.sa) \\ ${ }^{b}$ Department of Mathematics, Larbi Ben M'hidi University, Oum El Bouaghi, Algeria \\ (hzekraoui421@gmail.com)
}

Communicated by F. Lin

\begin{abstract}
The aim of this paper is to study some topological distances properties, semidendrites and convexity on the symmetrized omega algebra. Furthermore, some properties and exponents on the symmetrized omega algebra are introduced.
\end{abstract}

\section{MSC: 15A80; 16Y60; 54F65.}

KEYWORDS: omega algebra; symmetrized Omega algebra; semidendrite; exponents; convex and topology.

\section{INTRODUCTION}

Omega algebra unifies the min and the max plus algebras and introduces an original structure which in fact is an "abstract tropical algebra". termed it as "Omega algebra" or in short just, " $\omega$ - algebra". The $\mathbb{R}_{-\infty}$ and $\mathbb{R}_{\infty}$ and their nearby structures, like min - max and max - times algebras, etc., are all subsumed under Omega algebra. All these are idempotent semirings which sometimes also termed as dioids. In the previous studies, for the construction of all such semirings, an ordered infinite abelian group is mandatory, see [4], [5], [7] and [8]. In $\omega$ - algebra, the definition is extended to cyclically ordered abelian groups and also for finite sets under some suitable ordering, for more details one can refer to [3]. 
The aim of this paper is to define some topological distances, semidendrites, convexity, some properties and exponents on the symmetrized Omega algebra. Our paper is organized as follows. In Section 2, we review some basic facts for Omega algebra and a brief of the symmetrized Omega algebra, furthermore the rules of calculation and the absolute value on the symmetrized Omega algebra. In Section 3, some properties and exponents on the symmetrized Omega algebra are introduced. We study some properties of the given topological distances and semidendrite on the symmetrized Omega algebra in Section 4. In Section 5 , we generalize the notion of convex sets in paper [6] over $\mathbb{S}_{\omega}$. This paper is produced from the $\mathrm{PhD}$ thesis of Mr. Mesfer Hayyan Alqahtani in King Abdulaziz University..

\section{Some Preliminaries in abstract Omega Algebra}

In this section, we recall some basic facts for Omega algebra, the symmetrized Omega algebra, rules of calculation and $\omega$-absolute value. For more details see [3].

2.1. Omega algebra. Let $(G, \circ, e)$ be an abelian group. Let $A$ be a closed subset of $G$ and $e \in A$. Then $(A, \circ, e)$ is a submonoid of $G$. Assume that $\omega$ is an indeterminate (may belong to $A$ or $G$, as we will see in Examples 1 and 2 . Obviously, in this case $\omega$ is no longer an indeterminate). Because the terms are generated from tropical geometry, so such an indeterminate may be termed as a tropical indeterminate.

Definition 2.1 ([3]). We say that $A_{\omega}=A \cup\{\omega\}$ is an Omega algebra (in short $\omega$-algebra) over the group $G$ in case $A_{\omega}$ is closed under two binary operations,

$$
\oplus, \otimes: A_{\omega} \times A_{\omega} \longrightarrow A_{\omega},
$$

such that $\forall a_{1}, a_{2}, a_{3} \in A$, the following axioms are satisfied:

1) $a_{1} \oplus a_{2}=a_{1}$ or $a_{2}$;

2) $a_{1} \oplus \omega=a_{1}=\omega \oplus a_{1}$

3) $\omega \oplus \omega=\omega$;

4) $a_{1} \otimes a_{2}=a_{2} \otimes a_{1} \in A$;

5) $\left(a_{1} \otimes a_{2}\right) \otimes a_{3}=a_{1} \otimes\left(a_{2} \otimes a_{3}\right)$;

6) $a_{1} \otimes e=a_{1}$;

7) $a_{1} \otimes \omega=\omega \otimes a_{1}=\left\{\begin{array}{ccc}\omega & \text { if } & \omega \neq e \\ a_{1} & \text { if } & \omega=e\end{array}\right.$;

8) $\omega \otimes \omega=\omega$

9) $a_{1} \otimes\left(a_{2} \oplus a_{3}\right)=\left(a_{1} \otimes a_{2}\right) \oplus\left(a_{1} \otimes a_{3}\right)$.

Remark 2.2 ([3]).

1) $\oplus$ is a pairwise comparison operation, such as, max, min, inf, sup, up, down, lexicographic ordering, or any thing else that compairs two elements of $A_{\omega}$. Obviously, it is associative and commutative and the tropical indeterminate $\omega$ plays the role of the identity. Hence $\left(A_{\omega}, \oplus, \omega\right)$ is a commutative monoid. 
$2) \otimes$ is also associative and commutative on $A_{\omega}$ and $e$ plays the role of the multiplicative identity of $A_{\omega}$. Hence $\left(A_{\omega}, \otimes, e\right)$ is also a commutative monoid.

3) The left distributive law (9) also gives the right distributive law.

4) Every element of $A_{\omega}$ is an idempotent under $\oplus$.

5) Altogether, we write both structures as: $A_{\omega}=\left(A_{\omega}, \oplus, \otimes, \omega, e\right)$. This is an idempotent semiring also called "dioid" in literature.

Remark 2.3 ([3]). In this note, we confined ourselves to only $\omega$ - algebras over abelian groups and rings.

2.2. The symmetrized Omega algebra. In this subsection, we give a brief of the symmetrized Omega algebra, for more details see [3].

Let $(G, \circ, e)$ be an abelian group and $\left(A_{\omega}, \oplus, \otimes, \omega, e\right)$ an $\omega$-algebra over the group $G$. We consider the set of ordered pairs $\mathcal{P}_{\omega}=A_{\omega}^{2}$. Let $\leq$ be the ordering defined on $A_{\omega}$ by the relation

$$
a \leq b \Longleftrightarrow a \oplus b=b
$$

which gives a total order on $A_{\omega}$ and for all $a \in A_{\omega}$, we have $\omega \leq a$. For $a \neq b$, such that $a \oplus b=b$, we denote by $a<b$. Let $\nabla$ be the relation defined on $\mathcal{P}_{\omega}$ as follows: for all $(a, b),(c, d) \in \mathcal{P}_{\omega}$

$$
(a, b) \nabla(c, d) \Longleftrightarrow a \oplus d=b \oplus c .
$$

Definition 2.4 ([3]). Let $\sim$ be the equivalence relation close to $\nabla$ defined as follows: for all $(a, b),(c, d) \in \mathcal{P}_{\omega}$,

$$
(a, b) \sim(c, d) \Longleftrightarrow \begin{cases}(a, b) \nabla(c, d) & \text { if } a \neq b \text { and } c \neq d \\ (a, b)=(c, d) & \text { otherwise }\end{cases}
$$

In addition to the class element $\bar{\omega}=\overline{(\omega, \omega)}$; for all $a \in A_{\omega}$, with $a \neq \omega$, we have three kinds of equivalence classes:

(a) $\overline{(a, \omega)}=\left\{(a, b) \in \mathcal{P}_{\omega}, b<a\right\}$, called positive $\omega$-element.

(b) $\overline{(\omega, a)}=\left\{(b, a) \in \mathcal{P}_{\omega}, b<a\right\}$, called negative $\omega$-element.

(c) $\overline{(a, a)}$ called balenced $\omega$-element.

Proposition $2.5([3])$. The addition operation $\bar{\oplus}$ defined by

$$
\overline{(a, b)} \overline{\oplus(c, d)}=\overline{(a \oplus c, b \oplus d)}
$$

on the quotient set $\frac{\mathcal{P}_{\omega}}{\sim}$ is well defined and satisfies the axioms (1), (2) and (3) of Definition 2.1.

Proposition 2.6 ([3]). The set $\frac{\mathcal{P}_{\omega}}{\sim}$ is closed under the binary multiplication operation $\bar{\otimes}$ defined as follows:

for all $\overline{(a, b)}, \overline{(c, d)} \in \frac{\mathcal{P}_{\omega}}{\sim}$;

$$
\overline{(a, b)} \bar{\otimes} \overline{(c, d)}=\overline{((a \otimes c) \oplus(b \otimes d),(a \otimes d) \oplus(b \otimes c))}
$$

and satisfies axioms from (4) to (9) of Definition 2.1 with the unit class element $\bar{e}=\overline{(e, \omega)}$. 
Definition 2.7 ([3]). The structure $\left(\frac{\mathcal{P}_{\omega}}{\sim}, \bar{\oplus}, \bar{\otimes}, \bar{\omega}, \bar{e}\right)$ is called the symmetrized $\omega$-algebra over the abelian group $G \times G$ and denoted by $\mathbb{S}_{\omega}$.

In the coming sections just for simplicity we will use $\oplus, \otimes, \omega$ and $e$ instead of $\bar{\oplus}, \bar{\otimes}, \bar{\omega}$ and $\bar{e}$ respectively.

Remark 2.8 ([3]).

1) Despite the nature of the positive and the negative $\omega$-elements, they are not the inverses of each others for the additive operation $\bar{\oplus}$,

2) We have three symmetrized $\omega$-subalgebras of $\mathbb{S}_{\omega}$,

$$
\begin{aligned}
& \mathbb{S}_{\omega}^{(+)}=\left\{\overline{(a, \omega)}, a \in A_{\omega}\right\}, \\
& \mathbb{S}_{\omega}^{(-)}=\left\{\overline{(\omega, a)}, a \in A_{\omega}\right\}, \\
& \mathbb{S}_{\omega}^{(0)}=\left\{\overline{(a, a)}, a \in A_{\omega}\right\} .
\end{aligned}
$$

3) The three symmetrized $\omega$-subalgebras of $\mathbb{S}_{\omega}$ are connected by the zero class element $\bar{\omega}$.

4) The positive $\omega$-elements and the negative $\omega$-elements are called signed and denoted by $\mathbb{S}_{\omega}^{\vee}=\mathbb{S}_{\omega}^{(+)} \cup \mathbb{S}_{\omega}^{(-)}$, where the zero class $\overline{(\omega, \omega)}$ corresponds to $\omega$.

2.3. Rules of calculation in Omega. Let $a \in A_{\omega}$. Then we admit the following notations:

$$
+a=\overline{(a, \omega)},-a=\overline{(\omega, a)}, \cdot a=\overline{(a, a)} .
$$

By results in Proposition 2.5, Proposition 2.6 and the above notation, it is easy to verify the rules of calculation in the following proposition:

Proposition 2.9 ([3]). For all $a, b \in A_{\omega}$, we have

$$
\begin{aligned}
& \text { (i) }(+a) \oplus(+b)=+(a \oplus b) \text {; } \\
& \text { (ii) }(+a) \oplus(-b)=\left\{\begin{array}{ll}
+a & \text { if } b<a \\
-b & \text { if } b>a \\
\cdot a & \text { if } b=a
\end{array}\right. \text {; } \\
& \text { (iii) }( \pm a) \oplus(\cdot b)=\left\{\begin{array}{ll} 
\pm a & \text { if } b<a \\
\cdot b & \text { if } b>a
\end{array}\right. \text { : } \\
& \text { (iv) }(-a) \oplus(-b)=-(a \oplus b) \text {; } \\
& \text { (v) }(+a) \otimes(+b)=+(a \otimes b) \text {; } \\
& \text { (vi) }(+a) \otimes(-b)=-(a \otimes b) \text {; } \\
& \text { (vii) }( \pm a) \otimes(\cdot b)=\cdot(a \otimes b) \text {; } \\
& \text { (viii) }(-a) \otimes(-b)=+(a \otimes b) \text {. }
\end{aligned}
$$

From the previous rules, we can notice that the sign of the result in the addition operation follows the greater element in $A_{\omega}$. While in the multiplication operation, the balance sign is the strong one (has priority).

From Proposition 2.9, we can deduce the following : 
Proposition $2.10([3])$. The map $|\cdot|_{\omega}: \mathbb{S}_{\omega} \longrightarrow A_{\omega}$, such that for all $a \in A_{\omega}$,

$$
|+a|_{\omega}=|-a|_{\omega}=|\cdot a|_{\omega}=a
$$

is an absolute value on $\mathbb{S}_{\omega}$. We call it the $\omega$-absolute value.

\section{Some properties And exponents on $\mathbb{S}_{\omega}$}

In this section, we give some properties and exponents on $\mathbb{S}_{\omega}$.

3.1. Some properties on $\mathbb{S}_{\omega}$. As $A_{\omega}$ is isomorphic to the subdioid of pairs $\overline{(a, \omega)}, a \in A_{\omega}$, then $A_{\omega}$ itself can be considered as a subdioid of $\mathcal{P}_{\omega}$. If $u=\overline{(a, b)} \in \mathbb{S}_{\omega}$, then we have two unary operators: $\ominus$ (the $\omega$-algebraic minus operator) and ()$^{\bullet}$ (the balance operator) such that $\ominus u=\overline{(b, a)}$ and $u^{\bullet}=u \oplus(\ominus u)$.

Proposition 3.1. For all $u=\overline{(a, b)}, v=\overline{(c, d)} \in \mathbb{S}_{\omega}$, we have:

(i) $u^{\bullet}=(\ominus u)^{\bullet}=\left(u^{\bullet}\right)^{\bullet}$;

(ii) $u \otimes v^{\bullet}=(u \otimes v)^{\bullet}=u^{\bullet} \otimes v=u^{\bullet} \otimes v^{\bullet}$;

(iii) $\ominus(\ominus u)=u, \ominus(u \oplus v)=(\ominus u) \oplus(\ominus v)$ and $(\ominus u) \otimes v=\ominus(u \otimes v)=u \otimes(\ominus v)$.

Proof. A direct calculations gives the desired results.

Proposition 3.2. Let $a, b \in A_{\omega}$ be arbitrary. Then

1) $\ominus(+a)=-a$;

2) $\ominus(-a)=+a$;

3) $\ominus(\cdot a)=\cdot a$;

4) $+a \ominus+b=\overline{(a, b)}$.

Proof. 1) $\ominus(+a)=\ominus \overline{(a, \omega)}=\overline{\ominus(a, \omega)}=\overline{(\omega, a)}=-a$;

2) $\ominus(-a)=\ominus \overline{(\omega, a)}=\overline{\ominus(\omega, a)}=\overline{(a, \omega)}=+a$;

3) $\ominus(\cdot a)=\ominus \overline{(a, a)}=\overline{\ominus(a, a)}=\overline{(a, a)}=\cdot a$;

4) $+a \ominus+b=\overline{(a, \omega)} \ominus \overline{(b, \omega)}=\overline{(a, \omega)} \oplus \overline{(\overline{\ominus(b, \omega)}})=\overline{(a, \omega)} \oplus \overline{(\omega, b)}=\overline{(a, b)}$.

One can easily prove that the ordering $\leq$ defined on $A_{\omega}$ by the relation

$$
a \leq b \Longleftrightarrow a \oplus b=b
$$

is a total order on $A_{\omega}$ and for all $a \in A_{\omega}$, we have $\omega \leq a$. For $a \neq b$, such that $a \oplus b=b$, we denote by $a<b$. By the construction of the symmetrized Omega algebra, it is easy to extend the total order $\leq$ on $A_{\omega}$ to the ordering:

Definition 3.3. For any $u=\overline{(a, b)}, v=\overline{(c, d)} \in \mathbb{S}_{\omega}$, we have $u \leq v \Longleftrightarrow u \oplus v=v$

Corollary 3.4. For any $\overline{(a, b)} \in \mathbb{S}_{\omega}$, we have $\overline{(\omega, \omega)} \leq \overline{(a, b)}$.

Proof. $\overline{(a, b)} \oplus \overline{(\omega, \omega)}=\overline{(a \oplus \omega, b \oplus \omega)}=\overline{(a, b)} \Longleftrightarrow \bar{\omega}=\overline{(\omega, \omega)} \leq \overline{(a, b)}$. 
Proposition 3.5. Let $\overline{(a, b)} \in \mathbb{S}_{\omega}$, where $a, b \in A_{\omega}$, then we have:

1) If $a>b$ then $\overline{(a, b)}=\overline{(a, \omega)}$.

2) If $a<b$ then $\overline{(a, b)}=\overline{(\omega, b)}$.

3) If $a=b$ then $\overline{(a, b)}=\overline{(a, a)}$ or $\overline{(a, b)}=\overline{(b, b)}$.

Proof. 1) Let $a>b(a \neq \omega$, because $\omega>b$ impossible $)$. Since $\overline{(a, \omega)}=$ $\left\{(a, b) \in \mathcal{P}_{\omega}, b<a\right\}$, then $(a, b) \in \overline{(a, \omega)}$. Also $(a, b) \in \overline{(a, b)}$, because $(a, b) \sim(a, b)$. Then we have $(a, b) \in \overline{(a, \omega)} \cap \overline{(a, b)}$. Hence $\overline{(a, b)}=\overline{(a, \omega)}$, because any two equivalence classes are disjoint or equal.

2) Let $a<b(b \neq \omega$, because $a<\omega$ impossible $)$. Since $\overline{(\omega, b)}=\left\{(a, b) \in \mathcal{P}_{\omega}, b>a\right\}$, then $(a, b) \in \overline{(\omega, b)}$. Also $(a, b) \in \overline{(a, b)}$, because $(a, b) \sim(a, b)$. Then we have $(a, b) \in \overline{(\omega, b)} \cap \overline{(a, b)}$. Hence $\overline{(a, b)}=\overline{(\omega, b)}$, because any two equivalence classes are disjoint or equal.

3) If $a=b$, then we have $\overline{(a, b)}=\overline{(a, a)}$ or $\overline{(a, b)}=\overline{(b, b)}$.

Proposition 3.6. Let $u=\overline{(a, \omega)}, v=\overline{(\omega, b)}, z=\overline{(c, c)} \in \mathbb{S}_{\omega}$, where $a, b, c \in A_{\omega}$ are arbitrary. Then we have

1) If $a \geq b$, then $u \oplus v=u \oplus(\ominus v)$;

2) If $a<b$, then $u \oplus(\ominus v)=(\ominus u) \oplus(\ominus v)$;

3) If $b \leq c$, then $v \oplus z=(\ominus v) \oplus z=v \oplus(\ominus z)=(\ominus v) \oplus(\ominus z)$;

4) If $b>c$, then $(\ominus v) \oplus(\ominus z)=(\ominus v) \oplus z$ and $v \oplus z=v \oplus(\ominus z)$.

Proof. 1) 3)

$$
u \oplus v=\overline{(a, \omega)} \oplus \overline{(\omega, b)}=\overline{(a \oplus \omega, \omega \oplus b)}=\overline{(a, b)}=\overline{(a, \omega)}
$$

and

$$
u \oplus(\ominus v)=\overline{(a, \omega)} \oplus \overline{(b, \omega)}=\overline{(a \oplus b, \omega)}=\overline{(a, \omega)}
$$

By Equations 3.2 and 3.3, we get $u \oplus v=u \oplus(\ominus u)$.

$$
\begin{aligned}
u \oplus(\ominus u)=\overline{(a, \omega)} \oplus \overline{(b, \omega)}=\overline{(a \oplus b, \omega)}=\overline{(b, \omega)} \\
\text { and } \\
(\ominus u) \oplus(\ominus v)=\overline{(\omega, a)} \oplus \overline{(b, \omega)}=\overline{(b, a)}=\overline{(b, \omega)}
\end{aligned}
$$

By Equations 3.4 and 3.5, we get $u \oplus(\ominus v)=(\ominus u) \oplus(\ominus v)$.

$$
(\ominus v) \oplus z=\overline{(b, \omega)} \oplus \overline{(c, c)}=\overline{(b \oplus c, c)}=\overline{(c, c)}
$$

$$
v \oplus(\ominus z)=\overline{(\omega, b)} \oplus \overline{(c, c)}=\overline{(c, b \oplus c)}=\overline{(c, c)}
$$


Topological distances and geometry over the symmetrized Omega algebra

$$
(\ominus v) \oplus(\ominus z)=\overline{(b, \omega)} \oplus \overline{(c, c)}=\overline{(b \oplus c, c)}=\overline{(c, c)}
$$

By Equations 3.6, 3.7, 3.8 and 3.9, we get $v \oplus z=(\ominus v) \oplus z=v \oplus(\ominus z)=$ 4) $(\ominus v) \oplus(\ominus z)$

$$
(\ominus v) \oplus z)=\overline{(b, \omega)} \oplus \overline{(c, c)}=\overline{(b \oplus c, c)}=\overline{(b, c)}=\overline{(b, \omega)}
$$

By Equations 3.10 and 3.11, we get $(\ominus v) \oplus(\ominus z)=(\ominus v) \oplus z$. Also

$$
\begin{aligned}
& \qquad v \oplus z=\overline{(\omega, b)} \oplus \overline{(c, c)}=\overline{(c, b \oplus c)}=\overline{(c, b)}=\overline{(\omega, b)} \\
& \text { and } \\
& \qquad v \oplus(\ominus z)=\overline{(\omega, b)} \oplus \overline{(c, c)}=\overline{(c, b \oplus c)}=\overline{(c, b)}=\overline{(\omega, b)}
\end{aligned}
$$

By Equations 3.12 and 3.13, we get $v \oplus z=v \oplus(\ominus z)$.

3.2. Exponents on $\mathbb{S}_{\omega}$. In this subsection, we assume that $\otimes \mid A=0$. Let $\operatorname{sign}(.) \in\{+,-, \cdot\}$.

Definition 3.7. By Proposition 2.9, we can define the $\omega$-power of an element in $\mathbb{S}_{\omega}$ as following:

Let $\operatorname{sign}(a) a \in \mathbb{S}_{\omega}$ be arbitrary, where $a \in A_{\omega}$ and $n \in \mathbb{Z}^{+}$, then

(1): If $a \neq \omega$.

$$
\begin{aligned}
(\operatorname{sign}(a) a)^{\otimes n} & =\underbrace{(\operatorname{sign}(a) a) \otimes \ldots \otimes(\operatorname{sign}(a) a)}_{n \text { times }} \\
& =\operatorname{sign}(.) \underbrace{(a \otimes \ldots \otimes a)}_{n \text { times }} \\
& =\operatorname{sign}(.) \underbrace{(a \circ \ldots \circ a)}_{n \text { times }},
\end{aligned}
$$

where $\operatorname{sign}()=.\left\{\begin{array}{ll}\operatorname{sign}(a) & \text { if } \operatorname{sign}(a) \in\{+, \cdot\} \text { or } \operatorname{sign}(a)=- \text { and } n \text { is odd } \\ + & \text { if } \operatorname{sign}(a)=- \text { and } n \text { is even }\end{array} ;\right.$

(2): If $a=\omega$, then $(\operatorname{sign}(\omega) \omega)^{\otimes n}=\operatorname{sign}(\omega) \omega$.

Example 3.8. Let $-a \in \mathbb{S}_{\omega}$, where $a \neq \omega$, then 
1)

$$
\begin{aligned}
(-a)^{\otimes 2}=\overline{(\omega, a)} \overline{2}^{\otimes 2} \overline{(\omega, a)} \otimes \overline{(\omega, a)}= & \overline{(\omega \otimes \omega) \oplus(a \otimes a),(\omega \otimes a) \oplus(a \otimes \omega))} \\
& =\overline{(a \otimes a, \omega)} \\
& =\overline{(a \circ a, \omega)} \\
& =+(a \circ a)
\end{aligned}
$$

2)

$$
\begin{aligned}
& (-a)^{\otimes 3}=\overline{(\omega, a)}^{\otimes 3}=\overline{(\omega, a)} \otimes \overline{(\omega, a)} \otimes \overline{(\omega, a)} \\
& =\overline{((\omega \otimes \omega) \oplus(a \otimes a),(\omega \otimes a) \oplus(a \otimes \omega))} \otimes \overline{(\omega, a)} \\
& =\overline{(a \otimes a, \omega)} \otimes \overline{(\omega, a)} \\
& =\overline{((a \otimes a \otimes \omega) \oplus(\omega \otimes a),(a \otimes a \otimes a) \oplus(\omega \otimes \omega))} \\
& =\overline{(\omega, a \otimes a \otimes a)} \\
& =\overline{(\omega, a \circ a \circ a)} \\
& =-(a \circ a \circ a)
\end{aligned}
$$

Theorem 3.9. Let $A_{\omega}=\left(A_{\omega}, \oplus, \otimes, \omega, e\right)$ be an $\omega$ - algebra over an abelian group $G=(G, \circ, e)$ and $\mathbb{S}_{\omega}=\left(\frac{A_{\omega} \times A_{\omega}}{\sim}, \bar{\oplus}, \bar{\otimes}, \bar{\omega}, \bar{e}\right)$ is the symmetrized $\omega$-algebra over the abelian group $G \times G$. Let $\otimes \mid A=0, \omega \neq e$ and $a \neq \omega$. Then the following are equivalent:

1) If $a \in(A, \circ, e)$, has an inverse in $A$, then

2) $a \in\left(A_{\omega} \backslash\{\omega\}, \otimes, e\right)$ has a multiplicativ inverse, and then

3) $+a,-a \in \mathbb{S}_{\omega} \backslash \mathbb{S}_{\omega}^{(0)}$ have a multiplicative inverse, but $\cdot a \in \mathbb{S}_{\omega}^{(0)}$ has no multiplicative inverse.

Proof. $(1 \Rightarrow 2)$ Let $a \in(A, \circ, e)$ be arbitrary, which has an inverse and denoted by $a^{-1}$, then $a \otimes a^{-1}=a \circ a^{-1}=e$. Hence $a^{\otimes-1}=a^{-1}$ is the multiplicative inverse of $a$ in $\left(A_{\omega} \backslash\{\omega\}, \otimes, e\right)$.

$(2 \Rightarrow 3) \quad$ Let $a \in A_{\omega}$ be arbitrary, where $a \neq \omega$ and $\omega \neq e$, which has a multiplicative inverse and denoted by $a^{\otimes-1}$, then

$$
\begin{aligned}
&(+a) \otimes\left(+a^{\otimes-1}\right)=\overline{(a, \omega) \otimes \overline{\left(a^{\otimes-1}, \omega\right)}}=\overline{\left(\left(a \otimes a^{\otimes-1}\right) \oplus(\omega \otimes \omega),(a \otimes \omega) \oplus\left(\omega \otimes a^{\otimes-1}\right)\right)} \\
&=\overline{\left(a \otimes a^{\otimes-1}, \omega\right)} \\
&=\overline{\left(a \circ a^{\otimes-1}, \omega\right)}=\overline{(e, \omega)}=\bar{e}
\end{aligned}
$$

then $+a^{\otimes-1}$ is a multiplicative inverse of $+a$ in $\mathbb{S}_{\omega}$ and

$$
\begin{aligned}
(-a) \otimes\left(-a^{\otimes-1}\right)=\overline{(\omega, a)} \otimes \overline{\left(\omega, a^{\otimes-1}\right)}= & \overline{\left((\omega \otimes \omega) \oplus\left(a \otimes a^{\otimes-1}\right),\left(\omega \otimes a^{\otimes-1}\right) \oplus(a \otimes \omega)\right)} \\
& =\overline{\left(a \otimes a^{\otimes-1}, \omega\right)} \\
& =\overline{\left(a \circ a^{\otimes-1}, \omega\right)}=\overline{(e, \omega)}=\bar{e}
\end{aligned}
$$


then $-a^{\otimes-1}$ is a multiplicative inverse of $-a$ in $\mathbb{S}_{\omega}$.

On the other hand suppose that $\cdot a \in \mathbb{S}_{\omega}^{(0)}$ has a multiplicative inverse $\overline{(x, y)}$, where $x, y \in A_{\omega}$, then

$$
\begin{aligned}
(\cdot a) \otimes \overline{(x, y)}=\overline{(a, a)} \otimes \overline{(x, y)}= & \overline{((a \otimes x) \oplus(a \otimes y),(a \otimes y) \oplus(a \otimes x))} \\
& =\overline{((a \circ x) \oplus(a \circ y),(a \circ y) \oplus(a \circ x))}=\overline{(e, \omega)}
\end{aligned}
$$

hence $(a \circ x) \oplus(a \circ y)=e$ and $(a \circ y) \oplus(a \circ x)=\omega$, thus contradiction (note that if $\overline{(x, y)}=\cdot a^{\otimes-1}$, then we have $\left(a \circ a^{\otimes-1}\right) \oplus\left(a \circ a^{\otimes-1}\right)=e$ and $\left(a \circ a^{\otimes-1}\right) \oplus\left(a \circ a^{\otimes-1}\right)=\omega$, thus condradiction $)$.

$(3 \Rightarrow 1) \quad$ Let $+a \in \mathbb{S}_{\omega}$ be arbitrary, where $a \neq \omega, \omega \neq e$ and the multiplicative inverse of $+a$ in $\mathbb{S}_{\omega}$ is $\overline{(x, y)}$, where $x, y \in A_{\omega}$, then we have:

$$
\begin{aligned}
(+a) \otimes \overline{(x, y)}=\overline{(a, \omega)} \otimes \overline{(x, y)}= & \overline{((a \otimes x) \oplus(\omega \otimes y),(a \otimes y) \oplus(\omega \otimes x))} \\
& =\overline{(a \otimes x, a \otimes y)} \\
& =\overline{(a \circ x, a \circ y)}=\overline{(e, \omega)}=\bar{e}
\end{aligned}
$$

then $a \circ x=e$ and $a \circ y=\omega$. Hence $x=a^{-1}$ is the multiplicative of $a$ in $(A, \circ, e)$.

Let $-a \in \mathbb{S}_{\omega}$ be arbitrary, where $a \neq \omega, \omega \neq e$ and the multiplicative inverse of $-a$ is $\overline{(x, y)}$, where $x, y \in A_{\omega}$, then we have:

$$
\begin{aligned}
(-a) \otimes \overline{(x, y)}=\overline{(\omega, a)} \otimes \overline{(x, y)}= & \overline{(\omega \otimes x) \oplus(a \otimes y),(\omega \otimes y) \oplus(a \otimes x))} \\
& =\overline{(a \otimes y, a \otimes x)} \\
& =\overline{(a \circ y, a \circ x)}=\overline{(e, \omega)}=\bar{e}
\end{aligned}
$$

then $a \circ y=e$ and $a \circ x=\omega$. Hence $y=a^{-1}$ is the multiplicative inverse of $a$ in $(A, \circ, e)$.

Corollary 3.10. $\left(A_{\omega} \backslash\{\omega\}, \otimes, e\right)$ is a group if and only if for any $+a$ or $-a \in \mathbb{S}_{\omega} \backslash \mathbb{S}_{\omega}^{(0)}$, where $\otimes \mid A=0, \omega \neq e$ and $a \neq \omega$ has a multiplicative inverse.

Theorem 3.11. In $\mathbb{S}_{\omega}$, with $n, m \in \mathbb{Z}^{+}, a \in A_{\omega}$ be arbitrary and $\otimes \mid A=0$, the following exponents hold:

1) $(+a)^{\otimes n} \otimes(+a)^{\otimes m}=(+a)^{\otimes(m+n)}$

2) $(\cdot a)^{\otimes n} \otimes(\cdot a)^{\otimes m}=(\cdot a)^{\otimes(m+n)}$

3) $(-a)^{\otimes n} \otimes(-a)^{\otimes m}=(-a)^{\otimes(m+n)}$

4) $\left((+a)^{\otimes n}\right)^{\otimes m}=(+a)^{\otimes(m \times n)}$

5) $\left((\cdot a)^{\otimes n}\right)^{\otimes m}=(\cdot a)^{\otimes(m \times n)}$

6) $\left((-a)^{\otimes n}\right)^{\otimes m}=(-a)^{\otimes(m \times n)}$ 
Proof. 1) Let $a \neq \omega$, then

$$
\begin{aligned}
(+a)^{\otimes n} \otimes(+a)^{\otimes m} & =+\underbrace{(a \circ \ldots \circ a)}_{n \text { times }} \otimes+\underbrace{(a \circ \ldots \circ a)}_{m \text { times }} \\
& =+(\underbrace{(a \circ \ldots \circ a)}_{n \text { times }} \otimes \underbrace{(a \circ \ldots \circ a)}_{m \text { times }}) \\
& =+(\underbrace{(a \circ \ldots \circ a)}_{n \text { times }} \circ \underbrace{(a \circ \ldots \circ a)}_{m \text { times }}) \\
& =+\underbrace{(a \circ \ldots \circ a)}_{n+m \text { times }} \\
& =(+a)^{\otimes(m+n)} .
\end{aligned}
$$

If $a=\omega$, then

$(+\omega)^{\otimes n} \otimes(+\omega)^{\otimes m}=+\omega \otimes(+\omega)=+\omega=(+\omega)^{\otimes(m+n)}$.

2) By direct calculation similar above, we obtain to the desired result.

3) We have three cases:

Case I) Let $a \neq \omega$ and $n, m$ are odd (note that $n+m$ is even), then

$$
\begin{aligned}
(-a)^{\otimes n} \otimes(-a)^{\otimes m} & =-\underbrace{(a \circ \ldots \circ a)}_{n \text { times }} \otimes-\underbrace{(a \circ \ldots \circ a)}_{m \text { times }} \\
& =+(\underbrace{(a \circ \ldots \circ a)}_{n \text { times }} \otimes \underbrace{(a \circ \ldots \circ a)}_{m \text { times }}) \\
& =+(\underbrace{((a \circ \ldots \circ a)}_{n \text { times }} \circ \underbrace{(a \circ \ldots \circ a)}_{m \text { times }}) \\
& =+\underbrace{(a \circ \ldots \circ a)}_{n+m \text { times }} \\
& =(-a)^{\otimes(m+n)}
\end{aligned}
$$

If $a=\omega$, then

$(-\omega)^{\otimes n} \otimes(-\omega)^{\otimes m}=-\omega \otimes(-\omega)=+\omega=(-\omega)^{\otimes(m+n)}$.

Case II) Let $a \neq \omega$ and $n, m$ are even (note that $n+m$ is even), then

$$
\begin{aligned}
(-a)^{\otimes n} \otimes(-a)^{\otimes m} & =+\underbrace{(a \circ \ldots \circ a)}_{n \text { times }} \otimes+\underbrace{(a \circ \ldots \circ a)}_{m \text { times }} \\
& =+(\underbrace{(a \circ \ldots \circ a)}_{n \text { times }} \otimes \underbrace{(a \circ \ldots \circ a)}_{m \text { times }}) \\
& =+(\underbrace{(a \circ \ldots \circ a)}_{n \text { times }} \circ \underbrace{(a \circ \ldots \circ a)}_{m \text { times }}) \\
& =+\underbrace{(a \circ \ldots \circ a)}_{n+m \text { times }} \\
& =(-a)^{\otimes(m+n)}
\end{aligned}
$$


If $a=\omega$, then

$(-\omega)^{\otimes n} \otimes(-\omega)^{\otimes m}=+\omega \otimes(+\omega)=+\omega=(-\omega)^{\otimes(m+n)}$.

Case III) Let $a \neq \omega, n$ is odd and $m$ is even (note that $n+m$ is odd and we get to the same result if $n$ is even and $m$ is odd), then

$$
\begin{aligned}
(-a)^{\otimes n} \otimes(-a)^{\otimes m} & =-\underbrace{(a \circ \ldots \circ a)}_{n \text { times }} \otimes+\underbrace{(a \circ \ldots \circ a)}_{m \text { times }} \\
& =-(\underbrace{(a \circ \ldots \circ a)}_{n \text { times }} \otimes \underbrace{(a \circ \ldots \circ a)}_{m \text { times }}) \\
& =-(\underbrace{(a \circ \ldots \circ a)}_{n \text { times }} \circ \underbrace{(a \circ \ldots \circ a)}_{m \text { times }}) \\
& =-\underbrace{(a \circ \ldots \circ a)}_{n+m \text { times }} \\
& =(-a)^{\otimes(m+n)}
\end{aligned}
$$

If $a=\omega$, then

$(-\omega)^{\otimes n} \otimes(-\omega)^{\otimes m}=-\omega \otimes(+\omega)=-\omega=(-\omega)^{\otimes(m+n)}$.

4) Let $a \neq \omega$, then

$$
\begin{aligned}
\left((+a)^{\otimes n}\right)^{\otimes m} & =(+\underbrace{(a \circ \ldots \circ a)}_{n \text { times }})^{\otimes m} \\
& =(+\underbrace{(a \circ \ldots \circ a)}_{n \text { times }} \otimes \ldots \otimes+\underbrace{(a \circ \ldots \circ a)}_{n \text { times }}) \\
& =+\underbrace{(\underbrace{(a \circ \ldots \circ a)}_{n \text { times }} \otimes \ldots \otimes \underbrace{(a \circ \ldots \circ a)}_{n \text { times }}}_{n \text { times }}) \\
& =+\underbrace{(\underbrace{(a \circ \ldots \circ a)}_{n \text { times }} \ldots \ldots \underbrace{(a \circ \ldots \circ a)}_{n \text { times }}}_{n \text { times }}) \\
& =+\underbrace{(a \circ \ldots \circ a)}_{n \times m \text { times }} \\
& =(+\underbrace{a)^{\otimes(m \times n)}}_{\text {times }}
\end{aligned}
$$

If $a=\omega$, then

$\left((+\omega)^{\otimes n}\right)^{\otimes m}=(+\omega)^{\otimes m}=+\omega=(+\omega)^{\otimes(m \times n)}$.

5) By direct calculation similar above, we obtain to the desired result.

6) By direct calculation similar above with respect to $n$ and $m$ which is even or odd, we obtain to the desired result. 


\section{Some topological Distances PROPERTies ON $\mathbb{S}_{\omega}$}

By the Euclidean and frobenius distances definitions on papers [6] and [3] respectively, we give some topological distances properties over $\mathbb{S}_{\omega}$.

We denote the Euclidean metric in $\mathbb{R}^{n}$ by $d_{e, n}$ or by $d_{e}$ when it clear what $n$ is. The Euclidean metric in $\mathbb{C}^{n}$ is the same Euclidean metric in $\mathbb{R}^{2 n}$. As we have already mentioned, $\mathbb{R}^{n}$ will be usually considered with its Euclidean metric $d_{e, n}$ and with the natural topology induced by $d_{e, n}$.

From Paper [3] we recall that, some metrics were first time introduced and some algebraic and topological properties were studied in the symmetrized max-plus-algebra [6]. Let $(G, \circ)$ be a finitely generated (or finite) abelian group and $A_{\omega}$ be an $\omega$-algebra over $G$ such that the restriction $\otimes \mid A=0$. By the fundamental theorem of finitely generated (or finite) abelian groups, the group $G$ is a direct sum (direct product) of its cyclic groups, which make it isomorphic to a direct sum of finite copies of the cyclic groups $\mathbb{Z}$ of integers with finite summands of quotients of $\mathbb{Z}$ or isomorphic to a direct sum of cyclic groups of the quotients $\mathbb{Z}_{q_{i}}$ of $\mathbb{Z}$ (in other words, according to the group is infinite or finite, there exist some natural numbers $m, r, q_{1}, \ldots, q_{r}$ such that $G \cong \mathbb{Z}^{m}$ or $G \cong \mathbb{Z}^{m} \oplus \mathbb{Z}_{q_{1}} \oplus \ldots \oplus \mathbb{Z}_{q_{r}}$ or $\left.G \cong \mathbb{Z}_{q_{1}} \oplus \ldots \oplus \mathbb{Z}_{q_{r}}\right)$. In all cases we can represent an element of $G$ by $n$-tuple of elements of $\mathbb{Z}$ for some natural number $n$ via that isomorphism. From this point of view, we will define metrics (or semimetrics) on our $\omega$-algebra via the distance in $\mathbb{Z}$. Let $\Phi$ be a such isomorphism. For any $a \in G$, there exists $n \in \mathbb{N}$ and there exist $\alpha_{1}, \ldots, \alpha_{n} \in \mathbb{Z}$ (also they can be the representatives of classes in $\mathbb{Z})$, such that $\Phi(a)=\left(\alpha_{1}, \ldots, \alpha_{n}\right)$. Let us extend $\Phi$ on $A_{\omega}$ as follows:

$$
\Phi(a)= \begin{cases}\left(\alpha_{1}, \ldots, \alpha_{n}\right) & \text { for } a \in A, n \in \mathbb{N} \\ 0 & \text { for } a=\omega\end{cases}
$$

4.1. The Euclidean distance on $\mathbb{S}_{\omega}$. In this section, we suppose that the positive integer $n$ is the number of the direct sum (direct product) of copies of the cyclic groups $\mathbb{Z}$ of integers and/or the direct sum of the cyclic groups of quotients of $\mathbb{Z}$, which is isomorphic to $G$, where $G$ be a finitely generated (or finite) abelian group and $A_{\omega}$ be an $\omega$-algebra over $G$ such that the restriction $\otimes \mid A=\circ$.

Definition 4.1. The embedding of $\mathbb{S}_{\omega}$ into $\mathbb{C}^{n}$ is the mapping $\varphi: \mathbb{S}_{\omega} \rightarrow \mathbb{C}^{n}$ defined in the following way: Let $\theta$ be a cube root of unity, say, $\theta=\frac{-1+\sqrt{3} i}{2}$. As elements of $\mathbb{S}_{\omega}$ are defined by three signs, then we can emerge from $\mathbb{S}_{\omega}{ }^{2}$ into $\mathbb{C}^{n}$ by the map $\varphi$ defined by: for all $a \in A$, and by (4.1) we have $\Phi(a)=$ $\left(\alpha_{1}, \alpha_{2}, \ldots, \alpha_{n}\right)$, such that $n \in \mathbb{N}$ and $\alpha_{1}, \alpha_{2}, \ldots, \alpha_{n} \in \mathbb{Z}$, then 


$$
\varphi(\operatorname{sign}(a) a)= \begin{cases}\theta\left(\exp \left(\alpha_{1}\right), \ldots, \exp \left(\alpha_{n}\right)\right), & \text { if } a \in A \text { and } \operatorname{sign}(a)=+, \\ \theta^{2}\left(\exp \left(\alpha_{1}\right), \ldots, \exp \left(\alpha_{n}\right)\right), & \text { if } a \in A \text { and } \operatorname{sign}(a)=-, \\ \left(\exp \left(\alpha_{1}\right), \ldots, \exp \left(\alpha_{n}\right)\right), & \text { if } a \in A \text { and } \operatorname{sign}(a)=\cdot, \\ (0, \ldots, 0), & \text { if } a=\omega .\end{cases}
$$

We shall consider the natural metric in $\mathbb{S}_{\omega}$ that is defined below:

Definition 4.2. Let $d_{1}: \mathbb{S}_{\omega} \times \mathbb{S}_{\omega} \longrightarrow \mathbb{R}$ be defined as follows:

$$
d_{1}(\operatorname{sign}(a) a, \operatorname{sign}(b) b)=d_{e, 2 n}(\varphi(\operatorname{sign}(a) a), \varphi(\operatorname{sign}(b) b))
$$

for all $\operatorname{sign}(a) a, \operatorname{sign}(b) b \in \mathbb{S}_{\omega}$, where $d_{e, 2 n}$ is the Euclidean distance of $\mathbb{R}^{2 n}$. The metric $d_{1}$ in $\mathbb{S}_{\omega}$ will be called the Euclidean distance in $\mathbb{S}_{\omega}$.

\section{Remark 4.3. (1)}

1) It is clear that the Euclidean distances in $\mathbb{S}_{\omega}$ is induce the natural topology of $\mathbb{S}_{\omega}$. As a topological space, we shall consider $\mathbb{S}_{\omega}$ only with its natural topology.

2) We have $\left(\mathbb{C}^{n}, d_{e, 2 n}\right)$ is a metric space and let $\varphi\left(\mathbb{S}_{\omega}\right) \subseteq \mathbb{C}^{n}$. then the subspace metric on $\varphi\left(\mathbb{S}_{\omega}\right)$ is defined by simply restricting the metric on $\mathbb{C}^{n}$ to points in $\varphi\left(\mathbb{S}_{\omega}\right)$. In other words, for any $\varphi(\operatorname{sign}(a) a), \varphi(\operatorname{sign}(b) b) \in$ $\varphi\left(\mathbb{S}_{\omega}\right)$, we define

$\rho(\varphi(\operatorname{sign}(a) a), \varphi(\operatorname{sign}(b) b))=d_{e, 2 n}(\varphi(\operatorname{sign}(a) a), \varphi(\operatorname{sign}(b) b))$.

Hence $\left(\varphi\left(\mathbb{S}_{\omega}\right), \rho\right)$ is a metric subspace of a metric space $\left(\mathbb{C}^{n}, d_{e, 2 n}\right)$.

Proposition 4.4. Let $\left(\mathbb{S}_{\omega}, d_{1}\right)$ and $\left(\varphi\left(\mathbb{S}_{\omega}\right), \rho\right)$ be two metrics spaces, then

1) The mapping $\varphi$ is an isometry from $\left(\mathbb{S}_{\omega}, d_{1}\right)$ onto $\left(\varphi\left(\mathbb{S}_{\omega}\right), \rho\right)$.

2) $\left(\mathbb{S}_{\omega}, d_{1}\right)$ is homeomorphic to $\left(\varphi\left(\mathbb{S}_{\omega}\right), \rho\right)$.

Proof. 1) Let $\operatorname{sign}(a) a, \operatorname{sign}(b) b \in \mathbb{S}_{\omega}$ be arbitrary, then

$$
\begin{array}{r}
\rho(\varphi(\operatorname{sign}(a) a), \varphi(\operatorname{sign}(b) b))=d_{e, 2 n}(\varphi(\operatorname{sign}(a) a), \varphi(\operatorname{sign}(b) b)) \\
=d_{1}(\operatorname{sign}(a) a, \operatorname{sign}(b) b) .
\end{array}
$$

Hence $\varphi$ is an isometry map.

2) Since the map $\varphi$ from $\left(\mathbb{S}_{\omega}, d_{1}\right)$ onto $\left(\varphi\left(\mathbb{S}_{\omega}\right), \rho\right)$ is an isometry, then $\varphi$ is homeomorphism map. Hence $\left(\mathbb{S}_{\omega}, d_{1}\right) \cong\left(\varphi\left(\mathbb{S}_{\omega}\right), \rho\right)$.

Corollary 4.5. Since the mapping $\varphi$ from $\left(\mathbb{S}_{\omega}, d_{1}\right)$ onto $\left(\varphi\left(\mathbb{S}_{\omega}\right), \rho\right)$ is an isometry, then $\left(\mathbb{S}_{\omega}, \tau_{d_{1}}\right) \cong\left(\varphi\left(\mathbb{S}_{\omega}\right), \tau_{\rho}\right)$, where $\tau_{d_{1}}$ and $\tau_{\rho}$ are the natural topologies induced by the usual metrics $d_{1}$ and $\rho$ respectively.

Let $X$ be a topological space. We recall that it is said that a point $c \in X$ disconnects $X$ between points $a, b \in X$ if there exists a pair $U, V$ of disjoint open sets in $X$ such that $X \backslash\{c\}=U \cup V$ and $a \in U$, while $b \in V$. The space $X$ is a dendrite if it is a continuum such that, for each pair $a, b$ of distinct points of $X$, there exists a point $c \in X$ which disconnects $X$ between points $a$ and $b$ or the space $X$ is continuum if $X$ is compact, connected and metric 
space. It is said that $X$ is a semicontinuum if, for each pair $a, b$ of points of $X$, there is a continuum $C$ in $X$ such that $a, b \in C$. Let us say that $X$ is a semidendrite if it is a semicontinuum.

Proposition 4.6. The topological space $\left(\mathbb{S}_{\omega}, \tau_{d_{1}}\right)$ is a semidendrite.

Proof. Since $\varphi\left(\mathbb{S}_{\omega}\right) \subset \mathbb{C}^{n}$, then we have $\varphi\left(\mathbb{S}_{\omega}\right)$ is a semidendrite. By Corollary 4.5 , we have $\left(\mathbb{S}_{\omega}, \tau_{d_{1}}\right)$ is a semidendrite.

4.2. The Frobenius distance on $\mathbb{S}_{\omega}$. We suppose that the group $G$ is a direct sum of its $n$ cyclic subgroups (then $G$ is a finitely generated group). Then for every $a \in A$, we have

$\Phi(a)=\left(\alpha_{1}, \alpha_{2}, \ldots, \alpha_{n}\right)$, such that $n \in \mathbb{N}$. Let $c_{a}$ be a right circulant matrix, such that the first row is $\left(\alpha_{1}, \alpha_{2} \ldots, \alpha_{n}\right)$, then

$$
c_{a}=\left(\begin{array}{ccccc}
\alpha_{1} & \alpha_{2} & \ldots & \alpha_{n-1} & \alpha_{n} \\
\alpha_{n} & \alpha_{1} & \ldots & \alpha_{n-2} & \alpha_{n-1} \\
\alpha_{n-1} & \alpha_{n} & \ldots & \alpha_{n-3} & \alpha_{n-2} \\
\vdots & \vdots & \ddots & \vdots & \vdots \\
\alpha_{3} & \alpha_{4} & \ldots & \alpha_{1} & \alpha_{2} \\
\alpha_{2} & \alpha_{3} & \ldots & \alpha_{n} & \alpha_{1}
\end{array}\right)
$$

Each row of this matrix is right cyclic shift of the row above it. As right circulant matrices are diagonalizable in a same basis, we can benefit from this property to define a metric on $\mathbb{S}_{\omega}$ by using Frobenius norm \|\|$_{F}$ (it is a matrix norm). Let $\theta$ be a cube root of unity, say, $\theta=\frac{-1+\sqrt{3} i}{2}$. As elements of $\mathbb{S}_{\omega}$ are defined by three signs, then we can emerge from $\mathbb{S}_{\omega}$ into the algebra of right circulant matrices by the map $\phi$ defined by: for all $a \in A_{\omega}$,

$$
\phi(\operatorname{sign}(a) a)= \begin{cases}\theta \exp \left(\left(c_{a}\right)\right), & \text { if } a \in A \text { and } \operatorname{sign}(a)=+, \\ \theta^{2} \exp \left(\left(c_{a}\right)\right), & \text { if } a \in A \text { and } \operatorname{sign}(a)=-, \\ \exp \left(\left(c_{a}\right)\right), & \text { if } a \in A \text { and } \operatorname{sign}(a)=\cdot, \\ (0)_{n \times n}, & \text { if } a=\omega,\end{cases}
$$

Where $(0)_{n \times n}$ is the zero right circulant matrix.

Let us recall the Frobenius norm, sometimes also called the Euclidean norm is a matrix norm of an $m \times n$ matrix $A$, defined as the square root of the sum of the absolute squares of its elements,

$$
\|A\|_{F}=\sqrt{\sum_{i=1}^{m} \sum_{j=1}^{n}\left|a_{i j}\right|^{2}}
$$

Let $C_{n \times n}(\mathbb{C})$, be the space of all right circulant matrices over $\mathbb{C}$, the definition of distance over $C_{n \times n}(\mathbb{C})$ give by

$$
d_{F}(A, B)=\|A-B\|_{F},
$$

where $A, B \in C_{n \times n}(\mathbb{C})$. Hence $\left(C_{n \times n}(\mathbb{C}), d_{F}\right)$ is a metric space. 
Corollary 4.7. The exponential function of right circulant matrix is right circulant matrix [1] and the multiplication scalar of right circulant matrix is right circulant matrix. Hence, we have $\phi\left(\mathbb{S}_{\omega}\right) \subset C_{n \times n}(\mathbb{C})$.

Corollary 4.8. The map $\phi: \mathbb{S}_{\omega} \rightarrow C_{n \times n}(\mathbb{C})$ is an embedding.

Definition 4.9. Let $d_{2}: \mathbb{S}_{\omega} \times \mathbb{S}_{\omega} \longrightarrow \mathbb{R}$ be defined by

$$
\begin{aligned}
d_{2}(\operatorname{sign}(a) a, \operatorname{sign}(b) b) & =d_{F}(\phi(\operatorname{sign}(a) a), \phi(\operatorname{sign}(b) b)) \\
& =\|\phi(\operatorname{sign}(a) a)-\phi(\operatorname{sign}(b) b)\|_{F}
\end{aligned}
$$

for all $\operatorname{sign}(a) a, \operatorname{sign}(b) b \in \mathbb{S}_{\omega}$, where $d_{F}$ is the Frobenius distance of the right circulant matrices $C_{n \times n}(\mathbb{C})$. The metric $d_{2}$ in $\mathbb{S}_{\omega}$ will be called the Omega Frobenius distance in $\mathbb{S}_{\omega}$.

Remark 4.10. Let $\left(C_{n \times n}(\mathbb{C}), d_{F}\right)$ be a metric space. let $\phi\left(\mathbb{S}_{\omega}\right)$ be a non-empty subset of the right circulant matrices space $C_{n \times n}(\mathbb{C})$. Then $\left.d_{F}\right|_{\phi\left(\mathbb{S}_{\omega}\right) \times \phi\left(\mathbb{S}_{\omega}\right)}$, the restriction of $d_{F}$ to $\phi\left(\mathbb{S}_{\omega}\right) \times \phi\left(\mathbb{S}_{\omega}\right)$ is a metric on $\phi\left(\mathbb{S}_{\omega}\right)$. Let us denoted for $\left.d_{F}\right|_{\phi\left(\mathbb{S}_{\omega}\right) \times \phi\left(\mathbb{S}_{\omega}\right)}$ by $\rho$. In other words, for any $\phi(\operatorname{sign}(a) a), \phi(\operatorname{sign}(b) b) \in \phi\left(\mathbb{S}_{\omega}\right)$, we define

$$
\rho(\phi(\operatorname{sign}(a) a), \phi(\operatorname{sign}(b) b))=d_{F}(\phi(\operatorname{sign}(a) a), \phi(\operatorname{sign}(b) b)) .
$$

Hence $\left(\phi\left(\mathbb{S}_{\omega}\right), \rho\right)$ is a metric subspace of a metric space $\left(C_{n \times n}(\mathbb{C}), d_{F}\right)$.

Proposition 4.11. Let $\left(\mathbb{S}_{\omega}, d_{2}\right)$ and $\left(\phi\left(\mathbb{S}_{\omega}\right), \rho\right)$ be two metrics spaces, then

1) The mapping $\phi$ is an isometry from $\left(\mathbb{S}_{\omega}, d_{2}\right)$ onto $\left(\phi\left(\mathbb{S}_{\omega}\right), \rho\right)$.

2) $\left(\mathbb{S}_{\omega}, d_{2}\right)$ is homeomorphic to $\left(\phi\left(\mathbb{S}_{\omega}\right), \rho\right)$.

Proof. 1) Let $\operatorname{sign}(a) a, \operatorname{sign}(b) b \in \mathbb{S}_{\omega}$ be arbitrary, then

$$
\begin{array}{r}
\rho(\phi(\operatorname{sign}(a) a), \phi(\operatorname{sign}(b) b))=d_{F}(\phi(\operatorname{sign}(a) a), \phi(\operatorname{sign}(b) b)) \\
=d_{2}(\operatorname{sign}(a) a, \operatorname{sign}(b) b) .
\end{array}
$$

Hence $\phi$ is an isometry map.

2) Since the map $\phi$ from $\left(\mathbb{S}_{\omega}, d_{2}\right)$ onto $\left(\phi\left(\mathbb{S}_{\omega}\right), \rho\right)$ is an isometry, then $\phi$ is homeomorphism map. Hence $\left(\mathbb{S}_{\omega}, d_{2}\right) \cong\left(\phi\left(\mathbb{S}_{\omega}\right), \rho\right)$.

Corollary 4.12. Since the mapping $\phi$ from $\left(\mathbb{S}_{\omega}, d_{2}\right)$ onto $\left(\phi\left(\mathbb{S}_{\omega}\right), \rho\right)$ is an isometry, then $\left(\mathbb{S}_{\omega}, \tau_{d_{2}}\right) \cong\left(\phi\left(\mathbb{S}_{\omega}\right), \tau_{\rho}\right)$, where $\tau_{d_{2}}$ and $\tau_{\rho}$ are the natural topologies induced by the Omega Frobenius distance $d_{2}$ and Frobenius distance $\rho$ respectively.

Proposition 4.13. Let $\left(\mathbb{S}_{\omega}, \tau_{d_{2}}\right)$ and $\left(C_{n \times n}(\mathbb{C}), \tau_{d_{F}}\right)$ be a topological space, then

$$
\phi:\left(\mathbb{S}_{\omega}, \tau_{d_{2}}\right) \rightarrow\left(C_{n \times n}(\mathbb{C}), \tau_{d_{F}}\right)
$$

is an embedding map.

Proof. By Corollary 4.12, we have the space $\mathbb{S}_{\omega}$ is homeomorphic with the subspace $\phi\left(\mathbb{S}_{\omega}\right)$ of $C_{n \times n}(\mathbb{C})$. Hence the map $\phi$ is embedding. 


\section{Convexity in Symmetrized Omega Algebra}

In this section, we generalize the notion of convex sets in paper [6] over $\mathbb{S}_{\omega}$. We suppose that $n \in \mathbb{Z}^{+}$is the number of the direct sum (direct product) of copies of the cyclic groups $\mathbb{Z}$ of integers and/or the direct sum of the cyclic groups of quotients of $\mathbb{Z}$, which is isomorphic to $G$, where $G$ be a finitely generated (or finite) abelian group and $A_{\omega}$ be an $\omega$-algebra over $G$ such that the restriction $\otimes \mid A=\circ$ and $\omega \notin A$. By $\omega$ - absolute value, we have the following corollaries:

Corollary 5.1. Each $\operatorname{sign}(a) a \in \mathbb{S}_{\omega}$ can be written as

$$
\operatorname{sign}(a) a=\operatorname{sign}(a)|\operatorname{sign}(a) a|_{\omega}
$$

where $\operatorname{sign}(a) \in\{+.-$.$\} .$

Corollary 5.2. One can write the formulas for actions in $\mathbb{S}_{\omega}$ :

$$
\operatorname{sign}(a) a \oplus \operatorname{sign}(b) b=\operatorname{sign}(a \oplus b)\left(|\operatorname{sign}(a) a|_{\omega} \oplus|\operatorname{sign}(b) b|_{\omega}\right)
$$

where,

$$
\operatorname{sign}(a \oplus b)= \begin{cases}\operatorname{sign}(a), & a>b, \\ \operatorname{sign}(b), & b>a, \\ \operatorname{sign}(a), & a=b, \operatorname{sign}(a)=\operatorname{sign}(b), \\ \cdot, & a=b, \operatorname{sign}(a) \neq \operatorname{sign}(b),\end{cases}
$$

and the multiplication table for signs as follow.

$$
(\operatorname{sign}(a)) \otimes(\operatorname{sign}(b))= \begin{cases}+, & \text { if } \operatorname{sign}(a)=\operatorname{sign}(b)=+ \text { or }-, \\ -, & \text { if } \operatorname{sign}(a) \neq \operatorname{sign}(b), \operatorname{sign}(a) \neq \cdot \text { and } \operatorname{sign}(b) \neq \cdot, \\ \cdot, & \text { if either } \operatorname{sign}(a)=\cdot \text { or } \operatorname{sign}(b)=\cdot,\end{cases}
$$

For any $\operatorname{sign}(a) a, \operatorname{sign}(b) b \in \mathbb{S}_{\omega}$, we can define $\operatorname{sign}(a) a \oplus \operatorname{sign}(b) b \in \mathbb{S}_{\omega}$ and $\operatorname{sign}(a) a \otimes \operatorname{sign}(b) b \in \mathbb{S}_{\omega}$ as in Equations 5.1 and 5.2 respectively. Then $\left(\mathbb{S}_{\omega}, \oplus, \otimes\right)$ is a semiring. define

By a scalar we mean an element of $A_{\omega}$. For $\operatorname{sign}(a) a \in \mathbb{S}_{\omega}, \mu \in A_{\omega}$, we

$$
\mu \otimes \operatorname{sign}(a) a=\operatorname{sign}(a)\left(\mu \circ|\operatorname{sign}(a) a|_{\omega}\right)
$$

The operation $\otimes: A_{\omega} \times \mathbb{S}_{\omega} \rightarrow \mathbb{S}_{\omega}$ is well defined, and we can consider it as an outer operation from $A_{\omega} \times \mathbb{S}_{\omega}$ to $\mathbb{S}_{\omega}$ or the restriction to $A_{\omega} \times \mathbb{S}_{\omega}$ of the inner operation $\otimes$ in $\mathbb{S}_{\omega}$ because $A_{\omega} \subset \mathbb{S}_{\omega}$. Considering $\otimes$ as the outer operation, we can look at $\mathbb{S}_{\omega}$ as at a semimodule("vector space over semiring") over the semiring $A_{\omega}$. 
We recall that, for each pair of points $x_{1}, x_{2}$ of a space $X$, a segment $\left[x_{1}, x_{2}\right]$ with end-points $x_{1}$ and $x_{2}$ is defined, then a set $A \subseteq X$ is called convex if, for all $x_{1}, x_{2} \in A$, we have $\left[x_{1}, x_{2}\right] \subseteq A$ see [2]. In particular, for $\operatorname{sign}(a) a, \operatorname{sign}(b) b \in \mathbb{S}_{\omega}$, we can consider distinct kinds of segments with endpoints $\operatorname{sign}(a) a$ and $\operatorname{sign}(b) b$, between them,traditional segments and semimodule segments. These kinds of segments in $\mathbb{S}_{\omega}$ lead to distinct kinds of convexity in $\mathbb{S}_{\omega}$. Let us turn our attention to traditional and semimodule convexity in $\mathbb{S}_{\omega}$.

A convex set in $\mathbb{C}^{n}$ is a set $B \subseteq \mathbb{C}^{n}$ such that, for each pair $x_{1}, x_{2} \in B$, the traditional segment $\left[x_{1}, x_{2}\right]$ in the space $\mathbb{C}^{n}$ is contained in $B$.

Definition 5.3. A set $B \subseteq \mathbb{S}_{\omega}$ will be called traditionally convex if $\varphi(B)$ is convex in $\mathbb{C}^{n}$.

Remark 5.4.

1) Let us notice that a nonempty set $B \subseteq \mathbb{S}_{\omega}$ is simultaneously compact and traditionally convex in $\mathbb{S}_{\omega}$ if and only if $B$ is a traditional segment in $\mathbb{S}_{\omega}$.

2) All traditionally convex subsets of $\mathbb{S}_{\omega}$ are connected.

5.1. Semimodule convex sets in $\mathbb{S}_{\omega}$. The semimodule structure of $\mathbb{S}_{\omega}$ allows us to think about semimodule convexity of subsets of $\mathbb{S}_{\omega}$. In analogy to conventional algebra, we define a semimodule segment $[\operatorname{sign}(a) a, \operatorname{sign}(b) b]_{s}$ with end-points $\operatorname{sign}(a) a \in \mathbb{S}_{\omega}$ and $\operatorname{sign}(b) b \in \mathbb{S}_{\omega}$ as follows:

$$
\begin{gathered}
{[\operatorname{sign}(a) a, \operatorname{sign}(b) b]_{s}=\left\{(\mu \otimes \operatorname{sign}(a) a) \oplus(\gamma \otimes \operatorname{sign}(a) b): \mu, \gamma \in A_{\omega},\right. \text { with }} \\
\mu \oplus \gamma=e\} .
\end{gathered}
$$

Definition 5.5. A set $B \subseteq \mathbb{S}_{\omega}$ is said to be semimodule convex if, for each pair $\operatorname{sign}(a) a, \operatorname{sign}(b) b$ of points of $B$, the semimodule segment $[\operatorname{sign}(a) a, \operatorname{sign}(b) b]_{s}$ is contained in $B$.

ACKNOWLEDGEMENTs. The authors would thank the referees for the valuable remarks and advices. 


\section{REFERENCES}

[1] A. C. F. Bueno, On the exponential function of right circulant matrices, International Journal of Mathematics and Scientific Computing 3, no. 2 (2013).

[2] L. Hörmander, Notions of convexity, Progress in Mathematics 127, Birkhäuser, BostonBasel-Berlin (1994).

[3] S. Khalid Nauman, C. Ozel and H. Zekraoui, Abstract Omega algebra that subsumes min and max plus algebras, Turkish Journal of Mathematics and Computer Science 11 (2019) 1-10.

[4] G. L. Litvinov, The Maslov dequantization, idempotent and tropical mathematics: a brief introduction, Journal of Mathematical Sciences 140, no. 3 (2007), 426-444.

[5] D. Maclagan and B. Sturmfels, Introduction to Tropical Geometry, Graduate Studies in Mathematics, vol. 161, American Mathematical Society, 2015.

[6] C. Ozel, A. Piekosz, E. Wajch and H. Zekraoui, The minimizing vector theorem in symmetrized max-plus algebra, Journal of Convex Analysis 26, no. 2 (2019), 661-686.

[7] J.-E. Pin, Tropical semirings, Idempotency (Bristol, 1994), 50-69, Publ. Newton Inst., vol. 11, Cambridge Univ. Press, Cambridge, 1998.

[8] I. Simon, Recognizable sets with multiplicities in the tropical semiring, in: Mathematical Foundations of Computer Science (Carlsbad, 1988), Lecture Notes in Computer Science, vol. 324, Springer, Berlin, 1988, pp. 107-120. 